\title{
Propuesta de un diseño metodológico para la administración estratégica de riesgos en el sector de las telecomunicaciones
}

\author{
Proposal of a Methodological Design for the Risk Strategic Management in the Telecommunications Sector \\ Proposta de um desenho metodológico para a administração estratégica de riscos no setor de telecomunicações
}

Óscar Orlando Gómez Pinto

ORCID: http://orcid.org/0000-0002-2571-6289

\author{
DOI: https://doi.org/10.11144/Javeriana.cc19-47.pdma \\ Redalyc: http://www.redalyc.org/articulo.oa? \\ $\mathrm{id}=151556994010$
}

Fecha de recepción: 27 Septiembre 2017 Fecha de aprobación: 22 Mayo 2018

\section{Resumen:}

El desarrollo tecnológico y los nuevos y mejores servicios en el sector de las telecomunicaciones, obligan a las organizaciones a identificar sus riesgos para minimizar los posibles impactos y aprovechar sosteniblemente las oportunidades del mercado. El objetivo de esta investigación se centra en el desarrollo de una metodología para determinar el impacto de los procesos sobre los objetivos de negocio, precisar los procesos estratégicos clave, cuantificar su incidencia, identificar y evaluar los riesgos inherentes y reducir el costo asociado a la toma de decisiones. El resultado es una propuesta para la administración de riesgos estratégicos, fundamentada en la Teoría General de Sistemas y el balanced scorecard, que buscan facilitar la labor gerencial, el análisis estratégico y el entendimiento de la dinámica de los modelos de negocio, con base en el análisis de riesgos y en la evaluación de los controles establecidos en las organizaciones.

Palabras clave: administración estratégica, balanced scorecard, eTOM, riesgo, toma de decisiones.

\section{Abstract:}

The technological development as well as the newer and better services in the telecommunications sector are demanding the organizations to identify the risks in order to minimize their potential impacts and take advantage of the market opportunities in a sustainable way. The purpose in this research focuses on developing a methodology intended to ascertain the impact of the processes on the business objectives, to specify the key strategic processes, to quantify their influence, to identify and assess the inherent risks, and to reduce the cost associated to the decision-making. The result is a proposal of strategic risk management, grounded on the General Systems Theory and the balanced scorecard, which is intended to make easier the managerial work, the strategic analysis and the understanding of the business model dynamics based on the risk analysis and the evaluation of those controls set in the organizations.

Keywords: strategic management, balanced scorecard, eTOM, risk, decision-making.

\section{Resumo:}

O desenvolvimento tecnológico e os novos e melhores serviços no setor de telecomunicações obrigam às organizações a identificar seus riscos para minimizar os possíveis impactos e aproveitar sustentavelmente as oportunidades do mercado. O objetivo desta pesquisa foca no desenvolvimento de uma metodologia para determinar o impacto dos processos sobre os objetivos de negócio, precisar os processos estratégicos chave, quantificar sua incidência, identificar e avaliar os riscos inerentes e reduzir o custo associado à tomada de decisões. $\mathrm{O}$ resultado é uma proposta para a administração de riscos estratégicos, fundamentada na Teoria Geral de Sistemas e o balanced scorecard, que visam facilitar a labor gerencial, a análise estratégica e o entendimento da dinâmica dos modelos de negócio, com base na análise de riscos e na avaliação dos controlos estabelecidos nas organizações.

Palavras-chave: administração estratégica, balanced scorecard, eTOM, risco, tomada de decisões.

\section{Introducción}

La gestión de riesgos estratégicos permite diseñar e implementar un enfoque sistémico y sistemático para administrar los riesgos estratégicos de una organización, el conjunto de eventos externos y las tendencias 
que pueden afectar el crecimiento y la preservación de valor de la compañía, en una dinámica de constantes cambios, tanto tecnológicos como regulatorios.

Esta dinámica no ha sido ajena a las empresas latinoamericanas, en las que el sector de las telecomunicaciones se ha desarrollado rápidamente como consecuencia de la globalización, el aumento de la competencia, los mercados emergentes, el surgimiento y la rápida e incesante evolución y mejoramiento de las tecnologías de la información y la comunicación, entre otros. Por tanto, las empresas del sector de las telecomunicaciones enfrentan el reto de estar en un medio con un alto nivel de competitividad, por lo que debe contarse con nuevos modelos y herramientas de administración tendientes a lograr una gestión integral y oportuna.

En el desarrollo de la investigación se identificó un vacío metodológico, ante la ausencia de un modelo estándar para el sector en mención que integre la identificación, la evaluación y la administración de los riesgos de cara a la estrategia de negocio y minimice el impacto de los posibles eventos a los que se enfrentan las organizaciones.

En consecuencia, la presente investigación es una propuesta metodológica para el diseño de un modelo de administración de riesgos, con base en las teorías de balanced scorecard y administración estratégica, que busca evaluar la gestión empresarial en términos del aseguramiento de los objetivos estratégicos de corto, mediano y largo plazos.

El resultado esperado es la materialización de una metodología capaz de identificar, clasificar y ponderar los riesgos del negocio de las telecomunicaciones, respecto al cumplimiento de las metas definidas; para esto se tendrán como premisas del modelo, el aseguramiento de la continuidad del negocio y el crecimiento organizacional sostenido

Se ha tomado como soporte o fundamento el modelo Enhanced Telecom Operations Map (eTOM), para asegurar la estandarización del modelo, ya que los procesos son la base de la definición y el análisis de riesgos; sin embargo, depende de cada organización la priorización que le asigne a cada uno de ellos, a través del modelo, dentro de la cadena de valor, en términos del aporte o la importancia estratégica de acuerdo con los objetivos propuestos.

El producto de la investigación corresponde a la propuesta metodológica para la definición del mapa estratégico de riesgos y el balanced scorecard (Kaplan y Norton, 1996), alineados con la plataforma estratégica por medio de la ponderación de los procesos e indicadores dentro del sistema, respecto a los riesgos y objetivos estratégicos del sistema.

\section{Marco teórico}

\section{Administración estratégica}

Mediante la administración estratégica se formulan, implementan y evalúan las decisiones que permiten a la organización alcanzar sus objetivos, lo que implica integrar la administración, el mercadeo, el aspecto financiero y contable, la producción y las operaciones, la investigación y el desarrollo, y las tecnologías de la información y la comunicación para obtener el éxito de la organización (David, 2003).

Daft y Marcic (2006) explican que este proceso consta de tres etapas, a saber:

1. Formulación de la estrategia, que consiste en construir la misión de la empresa, advertir las oportunidades y las amenazas externas, definir las fortalezas y debilidades, fijar objetivos a largo plazo, generar estrategias alternativas y elegir las estrategias concretas que se seguirán.

2. Implementación o desarrollo de una cultura que sostenga la estrategia, creación de una estructura organizacional eficaz, modificación de las actividades de comercialización, preparación de los 
presupuestos, elaboración y empleo de sistemas de información y conexión entre la remuneración de los empleados y los resultados de la organización.

3. Evaluación, realizada mediante las siguientes actividades: a) revisión de los factores internos y externos que son la base de las estrategias presentes; b) medición del desempeño; c) aplicación de acciones correctivas.

\section{Toma de decisiones}

La toma estratégica de decisiones, en manos de los directivos, consiste en determinar los objetivos, políticas y recursos de la empresa (Martínez, 2002); en cuanto a conocimientos, se encarga de la innovación de productos y de servicios, y de la distribución de la información dentro de la organización; para el control operativo, determina la forma de realizar las tareas específicas establecidas en la mediana y la alta gerencia.

\section{Toma de decisiones con apoyo cuantitativo}

Según Saaty (1994), como los juicios y los valores varían de un individuo a otro, para comprenderlos, cooperar con estos y actuar coordinadamente es necesario acudir al Proceso Analítico Jerárquico (Analytic Hierarchy Process, AHP, por sus siglas en inglés), que es una teoría general sobre juicios y valoraciones que proporciona escalas de razón que capturan la realidad percibida, diferente de una asignación y normalización arbitraria de números. Es decir, que se asienta en el paradigma según el cual la complejidad inherente a un problema de toma de decisión con criterios múltiples se puede resolver mediante la jerarquización de los problemas planteados.

El AHP se caracteriza porque el problema de decisión funciona bajo una estructura en la que el objetivo o meta ocupa el nivel superior, los criterios con base en los cuales se toma la decisión ocupan el nivel intermedio, y el nivel inferior está constituido por las posibles alternativas para evaluar (Figura 1).

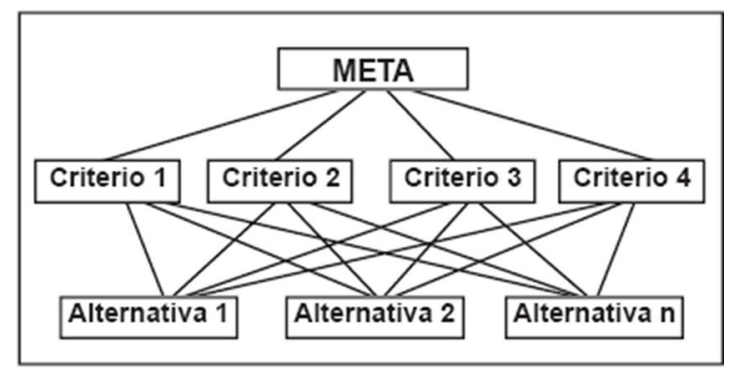

FIGURA 1.

Estructura del AHP.

Fuente: Vargas, R. (2010). Utilizando el proceso analítico jerárquico (PAJ) para seleccionar y priorizar proyectos en una cartera. Recuperado de: http://www.ricardo-vargas.com/articles/analytic-hierarchy-process/\#spanish

Entre pares de elementos de cada nivel jerárquico se hacen comparaciones cimentadas en la importancia o contribución de cada uno de ellos al elemento o nivel superior al que están vinculados. Mediante esta comparación se obtiene una escala de medida relativa de prioridades o pesos de los elementos en cuestión.

Cuando se comparan alternativas se emplean ratios de preferencia, en tanto que si se comparan criterios se emplean ratios de importancia. Lo relevante es que los pesos o prioridades relativas sumen uno.

En conclusión, el AHP es un modelo de decisión que interpreta la información gracias al establecimiento de juicios y medidas en una escala de razón dentro de una estructura jerárquica preestablecida, es decir, que se realiza la selección de alternativas en función de una serie de criterios o variables que por lo general se encuentran en conflicto (Saaty, 1997). 
Siguiendo a Saaty (1986), los axiomas que sirven de cimiento al AHP aparecen en la Tabla 1:

TABLA 1

Axiomas que fundamentan el AHP

\begin{tabular}{ll}
\hline Axioma & Descripción \\
\hline $\begin{array}{l}\text { Axioma de } \\
\text { comparación } \\
\text { recíproca }\end{array}$ & $\begin{array}{l}\text { Quien toma la decisión debe estar en capacidad de efectuar comparaciones } \\
\text { recíproca: "Si } A \text { es } x \text { veces preferido que } B \text {, entonces } B \text { es veces preferido } \\
\text { que } x 1 A \text { ". }\end{array}$ \\
Axioma de & Las preferencias se representan por medio de una escala limitada. \\
homogeneidad & Cuando se expresan preferencias se asume que los criterios son \\
Axioma & independientes de las propiedades de las alternativas. \\
de & \\
independencia & \\
Axioma de las & Para el propósito de la toma de una decisión se asume que la jerarquía es \\
expectativas & completa.
\end{tabular}

Fuente: elaboración propia, 2014.

La implementación del AHP, pasa por cuatro etapas (Martínez, 2007):

Primera etapa o etapa de modelización: aquí se construye una estructura jerárquica en la que queden representados todos los factores relevantes del proceso de resolución (actores, escenarios, factores, elementos e interdependencias), de tal modo que se conforma o constituye con un objetivo general y con los criterios. Debe señalarse que los elementos de un mismo nivel deben ser del mismo orden de magnitud y deben poder relacionarse con algunos o con todos los elementos del siguiente nivel.

Segunda etapa o etapa de valorización, en la que se integran las preferencias, gustos y deseos de los actores por medio de los juicios contenidos en las comparaciones que se hacen por pares, y muestran la preponderancia relativa de un elemento ante a otro respecto a un atributo o propiedad común, es decir, que se toma como unidad de referencia el elemento que posee el atributo en menor grado, y se pregunta con qué importancia, preferencia o verosimilitud el elemento que posee el atributo en mayor grado domina al otro. La comparación se hace por medio de una escala de valores que abarca un rango de 1 a 5 (Tabla 2 y Figura 2). 
TABLA 2

Escala de valores

\section{Escala numérica Escala verbal}

\begin{tabular}{ll}
\hline 1 & Ambos criterios o elementos son de igual importancia. \\
3 & Débil o moderada importancia de uno sobre el otro. \\
5 & Importancia absoluta de un criterio sobre otro. \\
\hline
\end{tabular}

Fuente: elaboración propia, 2013.

\begin{tabular}{|l|l|l|l|}
\cline { 2 - 4 } \multicolumn{1}{c|}{} & a & b & c \\
\hline a & 1 & 3 & 3 \\
\hline b & $1 / 3$ & 1 & 5 \\
\hline c & $1 / 3$ & $1 / 5$ & 1 \\
\hline
\end{tabular}

FIGURA 2.

Matriz de comparaciones pareadas.

Fuente: Saaty (1994a). How to Make a Decision: The Analytic Hierarchy Process. In: Interfaces, 24 (6), 19-43.

Luego de comparar los factores y de asignar los juicios de valor entre pares de factores, se hace el cálculo de peso (wj) para cada factor que puntualmente describe las características de los juicios de valor contemplados.

Entonces, para obtener el vector principal es preciso, en primera instancia, completar la matriz de comparación con los juicios de valor y posteriormente sumar cada columna (Figura 3):

\begin{tabular}{|l|l|l|l|}
\cline { 2 - 4 } \multicolumn{1}{c|}{} & a & b & $\mathrm{c}$ \\
\hline $\mathrm{a}$ & 1 & 3 & 3 \\
\hline $\mathrm{b}$ & $1 / 3$ & 1 & 5 \\
\hline $\mathrm{c}$ & $1 / 3$ & $1 / 5$ & 1 \\
\hline Total & 1,66 & 4,20 & 9 \\
\hline 1/Total & 0,60 & 0,24 & 0,11 \\
\hline
\end{tabular}

FIGURA 3.

Matriz de comparaciones

Fuente: Saaty (1994). How to Make a Decision: The Analytic Hierarchy Process. In: Interfaces, 24 (6), 19-43.

Posteriormente, para obtener el vector principal debe generarse una matriz auxiliar, de manera que en cada celda se anote el resultado que se obtenga tras dividir cada valor de juicio entre la sumatoria de la columna correspondiente, para luego promediar los valores normalizados de las filas, de modo que ese promedio refleja el vector principal.

Para los fines de esta investigación, el resultado obtenido se normaliza al sumar los valores en cada columna de la matriz de comparaciones pareadas, para luego establecer el inverso de cada columna y multiplicarlo por cada elemento de la matriz, lo que da como resultado una matriz de comparaciones normalizada. 
Tercera etapa: corresponde al análisis de las alternativas planteadas, para evaluar en qué medida satisfacen cada uno de los criterios. El establecimiento de la medida de satisfacción está en directa relación con las especificidades del criterio, por lo que puede determinarse bien sea a partir de una escala nominal, de una cardinal o de una ordinal. Acá se enfatiza en las prioridades tenidas en cuenta para la resolución del problema tratado, partiendo del hecho de que la prioridad se concibe como una unidad abstracta válida para cualquier escala, en la que se integran las preferencias que el individuo tiene al comparar aspectos tangibles e intangibles.

Cuarta etapa: es la síntesis del resultado con base en el aporte relativo de cada alternativa a cada uno de los criterios, así como del nivel de preferencia relativo asignado a ellos, de modo que se logre el objetivo general.

\section{Alineación estratégica}

Se refiere a la vinculación de las diversas unidades y departamentos de una empresa, incluyendo a cada uno de sus empleados, con la estrategia de la organización (misión, visión y valores), para que el trabajo cotidiano, el desempeño, las decisiones y el comportamiento estén directamente encaminados a apoyarla (Ruiz, Guzmán y Lluis de la Rosa i Esteva, 2007).

Su implementación se realiza de conformidad con el modelo que muestra la Tabla 3: 
TABLA 3

Modelo de alineación estratégica basado en el Cuadro de mando

\begin{tabular}{|c|c|c|c|}
\hline & Objetivos de gestión & Aporte & Beneficios \\
\hline $\begin{array}{l}1 . \\
\text { Planeamiento } \\
\text { estratégico }\end{array}$ & $\begin{array}{l}\text { Planear las acciones } \\
\text { organizativas alineadas } \\
\text { a la visión y misión de } \\
\text { la empresa. }\end{array}$ & $\begin{array}{l}\text { Planeamiento } \\
\text { organizativo }\end{array}$ & $\begin{array}{l}\text { Contar con una } \\
\text { planificación que } \\
\text { permita conseguir } \\
\text { objetivos estratégicos. }\end{array}$ \\
\hline $\begin{array}{l}2 . \\
\text { Cuadro de } \\
\text { mando }\end{array}$ & $\begin{array}{l}\text { Efectuar una gestión } \\
\text { estratégica balanceada }\end{array}$ & $\begin{array}{l}\text { Liderazgo gerencial } \\
\text { efectivo }\end{array}$ & $\begin{array}{l}\text { Mejorar los resultados } \\
\text { financieros con base en } \\
\text { una gestión balanceada }\end{array}$ \\
\hline $\begin{array}{l}3 . \\
\text { Gestión del } \\
\text { conocimiento }\end{array}$ & $\begin{array}{l}\text { Optimizar recursos e } \\
\text { incorporar innovación } \\
\text { a procesos y resultados }\end{array}$ & $\begin{array}{l}\text { Generación de una } \\
\text { espiral de } \\
\text { conocimientos }\end{array}$ & $\begin{array}{l}\text { Convertir el } \\
\text { conocimiento en un } \\
\text { valor }\end{array}$ \\
\hline $\begin{array}{l}4 . \\
\text { Gestión del } \\
\text { talento }\end{array}$ & Liderar el mercado & $\begin{array}{l}\text { Atracción y retención } \\
\text { de los mejores } \\
\text { profesionales }\end{array}$ & $\begin{array}{l}\text { Generar creatividad e } \\
\text { innovación }\end{array}$ \\
\hline $\begin{array}{l}5 . \\
\text { Gestión de } \\
\text { competencias }\end{array}$ & $\begin{array}{l}\text { Conducir a la } \\
\text { organización al éxito }\end{array}$ & $\begin{array}{l}\text { Consecución de una } \\
\text { mayor competitividad } \\
\text { organizativa }\end{array}$ & $\begin{array}{l}\text { Orientar hacia el éxito } \\
\text { organizativo }\end{array}$ \\
\hline $\begin{array}{l}6 . \\
\text { Gestión de la } \\
\text { calidad }\end{array}$ & $\begin{array}{l}\text { Competir en mercados } \\
\text { globales }\end{array}$ & $\begin{array}{l}\text { Desarrollo de procesos } \\
\text { de gestión } \\
\text { fundamentados en } \\
\text { normas de excelencia }\end{array}$ & $\begin{array}{l}\text { Conseguir } \\
\text { competitividad global }\end{array}$ \\
\hline $\begin{array}{l}7 . \\
\text { Gestión del } \\
\text { capital } \\
\text { intelectual }\end{array}$ & $\begin{array}{l}\text { Contabilizar y } \\
\text { capitalizar las } \\
\text { perspectivas de gestión } \\
\text { de personas, clientes... }\end{array}$ & $\begin{array}{l}\text { Ampliación de la base } \\
\text { de activos }\end{array}$ & $\begin{array}{l}\text { Aumentar el valor } \\
\text { financiero de la } \\
\text { organización }\end{array}$ \\
\hline
\end{tabular}

Fuente: Linx Recursos Humanos (2012)

\section{Balanced Scorecard}

Es una herramienta que permite implementar la estrategia y la misión de la empresa, a partir de un conjunto de medidas de actuación, enfatizando en la consecución de objetivos financieros, incluyendo los inductores de 
actuación futura para el logro de esos objetivos y proporcionando una estructura para transformar la estrategia en acción. Por medio del diagrama causa-efecto se establecen las hipótesis estratégicas, mediante la secuencia si/entonces, permitiendo anticipar a futuro, cómo el negocio creará valor para los clientes; sin embargo, estas causalidades son solo hipótesis que no tienen en cuenta los riesgos financieros, operativos, de mercado o relativos a la seguridad informática; es aquí donde la administración de riesgos, de una manera más robusta y con una óptica más amplia, entraría a mejorar el modelo, definiendo gráficos de causalidad equilibrados, en los que se espera encontrar afectaciones de variables que actualmente resultan inimaginables, que influyan directamente en el modelo o que se consideran o considerarían irrelevantes. De esta forma, tal como lo define la hipótesis de esta investigación, la administración de riesgos podría ser una herramienta útil para darle mayor robustez al modelo de Norton y Kaplan.

En concreto, el balanced scorecard implica la ampliación de la visión del empresario, en el sentido de incluir medidas de su compañía con ópticas diferentes a la financiera, de manera que cuente con la posibilidad de alcanzar objetivos que no se restrinjan al ámbito económico (Estupiñán y Estupiñán, 2006).

Esto entraña una radical transformación en los sistemas de gestión y de control, ya que los activos intangibles son tenidos en cuenta porque constituyen el fundamento a partir del cual se valora y asegura el crecimiento de la empresa.

\section{eTOM}

Es una herramienta que permite a las empresas de telecomunicaciones organizar el trabajo y enmendar los errores operativos, eludiendo la repetición de tareas, ya que se fijan definiciones precisas en los roles, lo que se materializa en un mapeo de la compañía que incluye cómo se hace la vinculación con los proveedores, cuáles son los ciclos de vida de los productos y los procesos de gestión de la empresa; en consecuencia, los consumidores reciben un beneficio indirecto, ya que sus operaciones se tornan más organizadas, coordinadas y regularizadas (Álvarez, 2008).

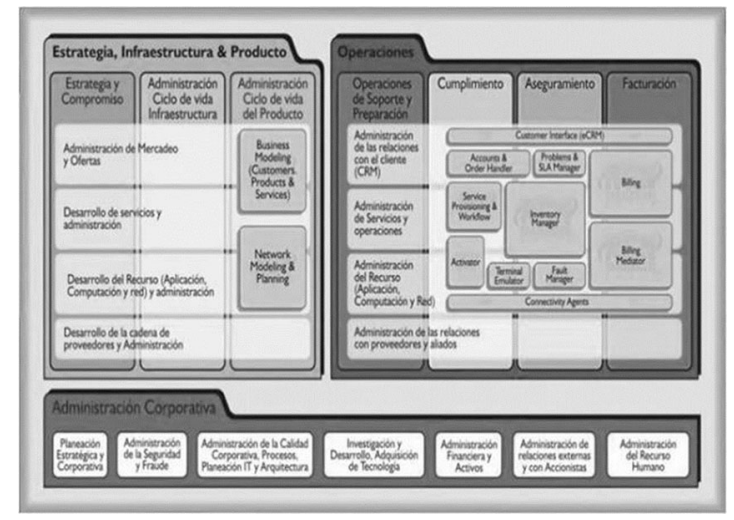

FIGURA 4.

Areas de procesos del eTOM

Fuente: IP Total Software S.A. (2008). Recuperado de http://www.iptotal.com/s_etom.html

\section{Riesgo}

La ISO/FDIS 31000 lo define como "el efecto de la incertidumbre sobre (el logro de) los objetivos".

El riesgo está caracterizado por la referencia a los eventos potenciales y a las consecuencias, o a una combinación de ellos; asimismo, se expresa en términos de una combinación de las consecuencias de un evento (incluyendo los cambios en las circunstancias) y en la probabilidad (Likelihood) de que suceda. 
Gestión de riesgos. Tiene que ver con la totalidad de esfuerzos que se hacen con el propósito de conocer el comportamiento de los entornos interno y externo de la organización, favoreciendo el decremento de la incertidumbre en cuanto al logro de los objetivos, por lo que se puede justificar si estos son o no alcanzables y por qué (Benavides, 2010).

Estos objetivos fijan el curso para lograr la alineación con los objetivos de la gestión de riesgos, lo que significa que, de manera general, buscan preservar la continuidad de las operaciones, y mantener una permanente mejora.

La gestión de riesgos debe operar como un todo bajo un plan de acción fundamentado en una priorización, tal como lo muestra la Figura 5:

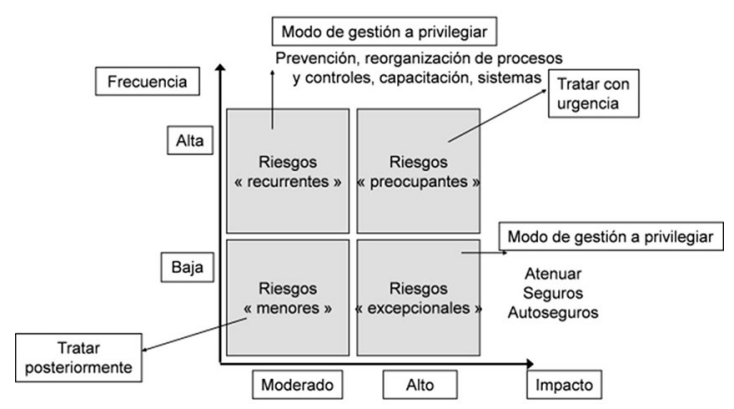

FIGURA 5.

Plan de acción de la gestión de riesgos fundamentado en la priorización.

Fuente: Ojeda. (2008). Por qué gestionar riesgos. Santiago de Chile: Instituto de Auditores Internos y Gobierno Corporativo.

\section{Modelo general propuesto}

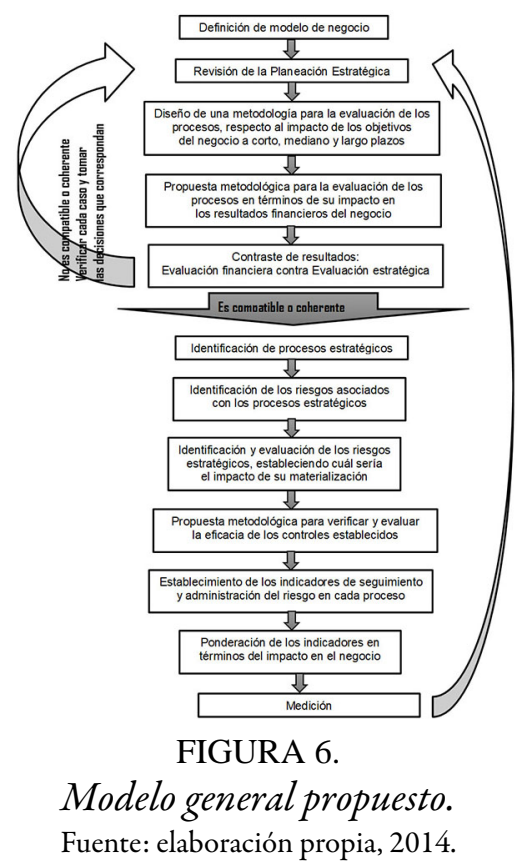

Para explicar el funcionamiento del modelo se desarrollarán cada una de las etapas propuestas, mediante su aplicación a un caso real empresarial.

Después de haber seleccionado la empresa que se va a intervenir se realizó la evaluación del modelo de negocio, lo que significó caracterizarla en cuanto a datos generales, historia, horizonte institucional, posición en el mercado, tarifas, etc. 
Seguidamente se revisó la planeación estratégica, se evaluaron los procesos, respecto al impacto de los objetivos del negocio a corto, mediano y largo plazos, lo que implicó emplear la escala numérica con la que los expertos califican los objetivos (Tabla 2); entonces se tomaron la misión y los objetivos para evaluarlos mediante una herramienta cuantitativa, se compararon con los demás y se asignaron los juicios de valor entre pares de factores, para calcular el peso para cada factor, describiendo las características de los juicios de valor contemplados.

Luego, para obtener el vector principal se generó una matriz auxiliar, de manera que en cada celda se anotó el resultado que se obtuvo tras dividir cada valor de juicio entre la sumatoria de la columna correspondiente, para luego promediar los valores normalizados de las filas, de modo que dicho promedio refleja el vector principal.

El resultado obtenido se normaliza sumando los valores en cada columna de la matriz de comparaciones pareadas, para establecer el inverso de cada columna y multiplicarlo por cada elemento de la matriz, de lo que resulta una matriz de comparaciones normalizada en la que obtenemos el peso relativo de cada proceso con respecto a la misión.

Para continuar con la evaluación de los procesos respecto a los objetivos del negocio, se procede de igual manera, como se hizo con la misión, con la visión.

El siguiente paso lo constituye la evaluación de los procesos, en términos de su impacto en los resultados financieros del negocio.

Para determinar los procesos clave o estratégicos relativos al proceso de información financiera, se sugiere desarrollar tal proceso anualmente.

Asimismo, se considera pertinente determinar la materialidad como el error máximo estimado en la información financiera; se debe asegurar que su ocurrencia no originará una situación de presentación de información engañosa a quienes estén interesados en la información.

El establecimiento de la materialidad deberá considerar aspectos cuantitativos y cualitativos; los primeros se relacionan con la existencia de aserciones falsas en los estados financieros que tienen un efecto cuantitativamente material, tanto individual como colectivo, en la información financiera, de ahí que su estipulación está en función de un componente crítico de los estados financieros.

Por su parte, los aspectos cualitativos para evaluar apuntan a considerar factores adicionales que pueden afectar la confiabilidad de los estados financieros.

Para calcular la materialidad es preciso determinar una referencia cuantitativa hacia el resto de los componentes de los estados financieros, lo que implica que debe ser un elemento estable sujeto de comparabilidad (ejemplos: utilidad antes de impuestos, utilidad neta, ingresos, total de activos, etc.).

Después de calcular la materialidad de cada cuenta en el balance y estado de resultados, esta se valida y se contrasta o mapea con los procesos de negocio, para que en este análisis se identifiquen los procesos materiales que deberán evaluarse en relación con sus riesgos inherentes.

Las aserciones financieras que tienen un efecto significativo en confiabilidad de las cuentas contables son relevantes. El objetivo de identificar aserciones sobre las cuentas contables significativas consiste en facilitar la determinación de los riesgos y controles que afectan la confiabilidad de la información financiera, por lo que deben ser consideradas como un referente permanente en el desarrollo de la evaluación del control interno.

Las aserciones financieras relevantes son las siguientes: existencia, integridad, valuación, asignación, derechos y obligaciones, y presentación y revelación.

Con referencia a la determinación del alcance de la evaluación del control interno, esta comienza con la identificación de cuentas significativas o de materiales a nivel de los estados financieros consolidados; se advierte que una cuenta es significativa si hay más de una probabilidad remota de que pudiera contener aserciones falsas que individualmente o en su agregado con otras podrían tener un efecto material en los estados financieros.

La determinación de las cuentas cualitativamente significativas se desarrolla teniendo en cuenta estos factores: complejidad y volumen de tracciones de la cuenta, naturaleza o composición de la cuenta, exposición 
a pérdidas por errores o fraude, probabilidad de contingencias significativas, cambios recientes en las características de la cuenta o de los procesos que la integran, alto grado de subjetividad y demás situaciones que incrementen la probabilidad de afectación de las aserciones contables.

Si la cuenta contable sujeta a evaluación contiene alguno de los anteriores factores, deberá asignarse el valor de uno (1) en el factor correspondiente, determinando como proceso crítico aquel que obtenga al menos una sumatoria de dos (2) en el factor determinado.

Seguidamente se hace el contraste de resultados de evaluación financiera contra evaluación estratégica, para jerarquizar los procesos, es decir, identificar los que dentro de la estrategia son de alta importancia y los que por su materialidad y probabilidad de aserciones falsas deben ser controlados estratégicamente.

De aquí se pasa a identificar los riesgos inherentes a los procesos estratégicos, se evalúa la eficacia y el costobeneficio de los controles existentes en cada proceso, estableciendo cuál sería el impacto financiero de su materialización, de modo que se obtengan las necesidades de nuevos controles estratégicos o de mejoramiento de los existentes; luego se consignan en una matriz en la que se proponen indicadores de gestión del riesgo, lo que da origen a un cuadro estratégico de control de riesgos.

\section{Propuesta metodológica de evaluación de controles estratégicos}

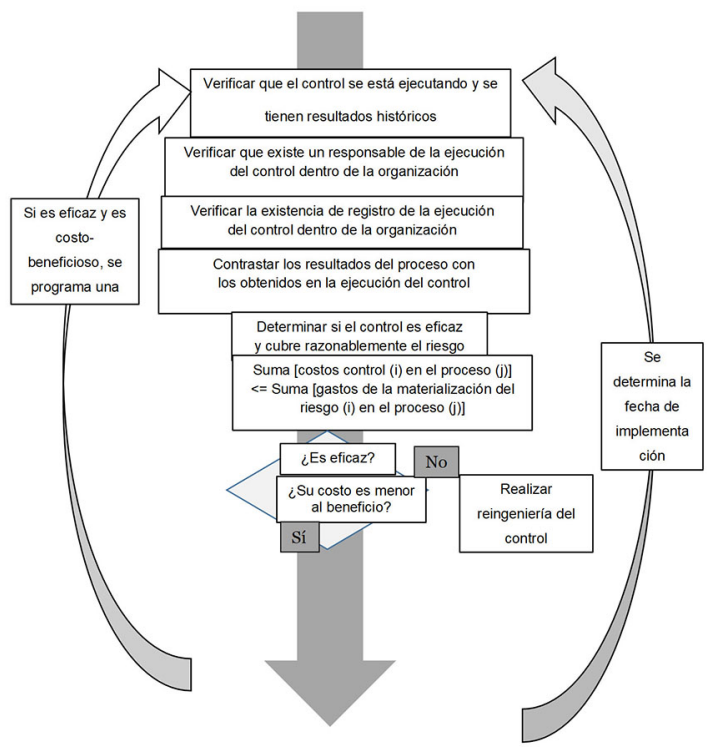

FIGURA 7.

Modelo general propuesto.

Fuente: elaboración propia, 2014.

En últimas, la presente propuesta metodológica se desarrolla mediante un proceso deductivo que minimiza la subjetividad, en razón del aprovechamiento de la alineación estratégica, del análisis mediante el empleo de las matrices y de la identificación de procesos estratégicos y controles por medición cuantitativa, con lo que realmente se consolida una efectiva y eficiente administración estratégica de riesgos en el sector de las telecomunicaciones.

En conclusión, la propuesta metodológica contribuiría, entre otros, a lo siguiente:

1. Generar coherencia entre la planeación estratégica y una adecuada gestión de riesgos estratégicos, identificando las áreas de negocio que impactan directamente sobre los objetivos de corto y largo plazos, definiendo controles estratégicos que contribuyan a la consecución de las metas establecidas. 
2. Minimizar la subjetividad del proceso de alineación estratégica por medio de la evaluación de cada uno de los procesos de negocio de acuerdo con su contribución a los planes de corto y largo plazos.

3. Reconocer oportunidades de negocio en un sector cambiante y en continua evolución tecnológica.

4. Enfocarse en los procesos de negocio que mueven los indicadores estratégicos propuestos.

5. Identificar y priorizar los recursos financieros destinados a la mitigación de riesgos.

\section{Referencias bibliográficas}

Álvarez, V. (2008). Modelo eTOM. Una ruta para ordenar los negocios. En: Perspectiva, 1 (1).

Benavides, L. (2010). Gestión de riesgos. Recuperado de: http://www.youtube.com/watch?v=YrpvwueYaAM

David, F. R. (2003). Conceptos de Administración Estratégica. México: Pearson Educación.

Daft, R. y Marcic, D. (2006). Introducción a la administración. México, D. F.: Cengage Learning Editores.

Estupiñán, R. y Estupiñán, O. (2006). Análisis financiero y de gestión. Bogotá: ECOE.

Kaplan, R. S. y Norton, D. P. (1996). The Balanced Scorecard: Translating Strategy Into Action. Boston: Harvard Business School Press.

Linx Recursos Humanos (2012). Modelo de Alineación Estratégica. Recuperado de http://www.linxrh.com/wordpre ss/modelo-de-alineacion-estrategica/

Martínez, E. (2007). Aplicación del proceso jerárquico de análisis en la selección de la localización de una PYME. En: Anuario Jurídico y Económico Escurialense, XV, 523-542.

Martínez, H. (2002). Toma de decisiones. Recuperado de: http://www.virtual.unal.edu.co/cursos/economicas/92402 /capitulos/capitulo10/cap107.html

Osterwalder, A y Pigneur, I. (2010). Business Model Generation. New Jersey, EE. UU.: John Wiley and Sons.

Pérez, A., Ramírez, C. y Rozo, J. (2012). Propuesta de un modelo de cuadro de mando integral para la ETB. Bogotá: EAN.

Ruiz, R. U., Guzmán, J. y Lluis de la Rosa i Esteva, J. (2007). Dirección empresarial asistida. Cómo alinear estratégicamente su organización. Madrid, España: Visión Net.

Saaty, T. L. (1997). Toma de decisiones para líderes: el proceso analítico jerárquico, la toma de decisiones en un mundo complejo. Oakville, Ontario, Canadá: RSW Publicaciones.

Saaty, T. L. (1994). Fundamentos de la toma de decisiones. Oakville, Ontario, Canadá: RSW Publicaciones.

Saaty, T. L. (1994a). How to Make a Decision: The Analytic Hierarchy Process. In: Interfaces, 24 (6), 19-43.

Saaty, T. L. (1986). Axiomatic Foundation of the Analytic Hierarchy Process. In: Management Science, 32(7), 841-855.

Licencia Creative Commons CC BY 4.0

Para citar este artículo: Gómez-Pinto, Ó. O. (2018). Propuesta de un diseño metodológico para la adminisn estratégica de riesgos en el sector de las telecomunicaciones. Cuadernos de Contabilidad, 19(47), 182-193. https://doi.org/10.11144/Javeriana.cc19-47.pdma 